\title{
The Research of The Method of Moving Object Detecting in Video Surveillance System
}

\author{
Hongxia $\mathrm{Wu}^{1}$
}

\begin{abstract}
Moving target detection is a very important in the intelligent monitoring system; however, the detection is still difficult in complex background. Therefore, the paper is based on the method of traditional background difference, we study that the video color information is taken as the detection of the characteristic quantity, the background is constantly and dynamically updated in order to match actual background more exactly with weight method, the threshold value is dynamically changed in Otsu method. The experimental results show that the algorithm achieves good detection results for complex environment.
\end{abstract}

Keywords: Intelligent monitoring, Moving object, the method of background difference, Threshold

\section{Introduction}

The intelligent video surveillance system based on computer vision has more and more widespread application, moving target detection is very important in the intelligent monitoring system, the image quality of moving target detected will affect its post-processing such as identification and classification of moving object, in the video surveillance system, the traditional detection methods of moving target includes optical flow method, the inter-frame difference and background difference. The independent moving targets can be detected by optical flow method, but it has high complexity of computation, big disturbance from noise and poor real-time. The inter-frame difference has low complexity and the shorter time between adjacent frames, therefore, the method is better suited detection in a dynamic environment, however, it is also easy to wrongly taken shaded background as target. The background difference is the most common method in the video surveillance system, but it is vulnerable to the impact of the three aspects. such as changing light, noise, the consistency of the updated background , the real background and the accuracy of the being selected threshold ${ }^{[1][2]}$. Therefore, the paper

\footnotetext{
${ }^{1}$ Hubei PolyTechnic University

416568921@QQ.com
} 
is based on the method of traditional background difference, we study that the video color information is taken as the detection of the characteristic quantity, the background is constantly and dynamically updated in order to match actual background more exactly with weight method, the threshold value is dynamically changed in Otsu method., which can achieve a good detection effect.

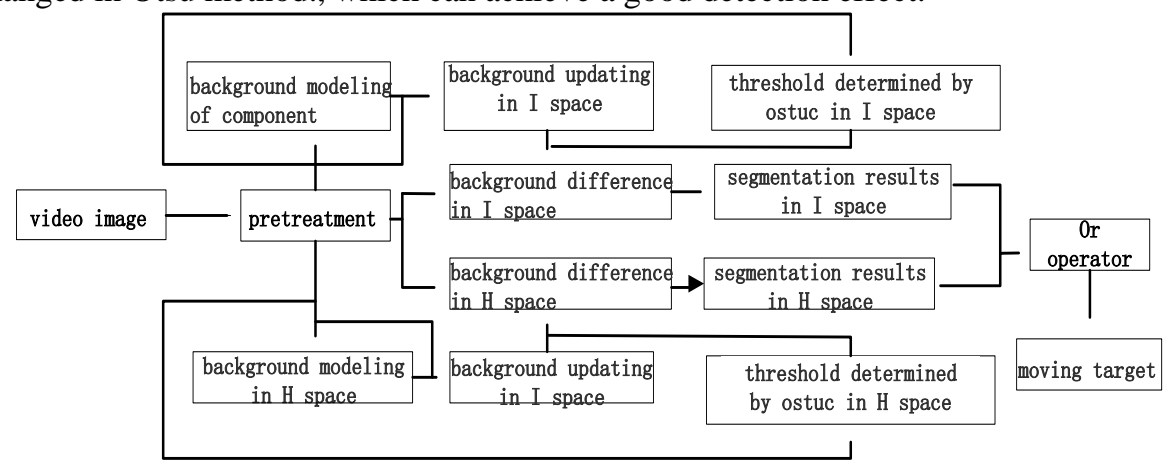

Fig. 1.1 the block diagram of moving target detection

\section{The detection of traditional background difference}

The detection of difference method has the inter-frame difference and background difference. The inter-frame difference is that moving target is obtained by subtracting the corresponding pixel values of two consecutive frames in the sequence of video images ${ }^{[3]}{ }^{[4]}$. The inter-frame difference has low complexity and the shorter time between adjacent frames, therefore, the method is better suited detection in a dynamic environment, however, it is also easy to wrongly taken shaded background as target. The background difference is able to provide a more accurate characteristic data compared to the inter-frame difference method, the probability is very small that is wrongly taken shaded background as target, because the background will be the impact of the dynamic factors of weather, light and tree jitter, the dynamic ideal background matching to the entire image frame is obtained, which can get a more accurate moving object ${ }^{[5][6]}$. Therefore, the paper is based on the method of traditional background difference, it is studied three aspects from the color space, background updating and threshold selecting, and the new solutions have proposed. The algorithm of background difference is briefly introduced as follows.

It is supposed that the current frame image is $f_{k}(x, y)$, the background frame image is $B_{k}(x, y)$, the corresponding difference image is shown in equation (2.1).

$$
D_{k}(x, y)=f_{k}(x, y)-B_{k}(x, y)
$$

When the differential image obtained is less than the threshold $\mathrm{T}$, the pixel is background, otherwise, the pixel is target, Principle is as shown in equation(2.2). a 
binary image is obtained according to the above principle, 0 is the background, 1 is object in the binary image.

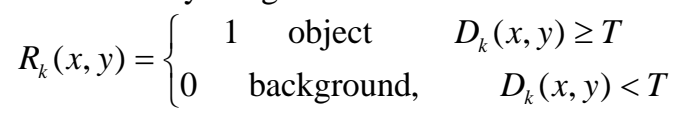

\section{The detection of background differ ence improved}

\subsection{The selection of color space}

The gray image is used to threshold segmentation by the detection of traditional background difference, the color images are captured in the most of the video surveillance system, and the part of the color information is lost in the process of the gray-scale image being processed, for example, the RGB values of the dark blue, brown and dark green are given in table 1, their gray values are calculated by the equation (3.1) in table 1,we can know from the calculation results that the different colored images have very similar gray in the gray conversion process of the color image. So that when it appears similar gray form the color change, moving targets can not detected using the gray value.

$$
\text { gray }=(R+G+B) / 3
$$

Table 1.1 RGB and gray of there color

\begin{tabular}{ccccc}
\hline color & $\mathrm{R}$ & $\mathrm{G}$ & $\mathrm{B}$ & gray \\
\hline dark green & 57 & 88 & 31 & 56 \\
dark blue & 25 & 44 & 82 & 50 \\
brown & 96 & 56 & 17 & 58 \\
\hline
\end{tabular}

Therefore, the color information is the key of threshold segmentation, the reasonable selecting of color space is the basis of selection of detection feature. HSI is most like human visual in various color space. In this paper, the color information is added to the detection of moving targets, equation (3.2),(3.3),(3.4) can convert the image from RGB space to HSI space.

$$
\begin{gathered}
H=\left\{\begin{array}{cl}
\arccos \left\{\frac{(R-G)+(R-B)}{2 \sqrt{(R-G)^{2}+(R-B)(G-B)}}\right\} \quad R \neq G \quad \text { or } \quad R \neq B \\
2 \pi-\arccos \left\{\frac{(R-G)+(R-B)}{2 \sqrt{(R-G)^{2}+(R-B)(G-B)}}\right\} & B>G
\end{array}\right. \\
S=1-\frac{3}{(R+G+B)} \min (R+G+B) \\
I=(R+G+B) / 3
\end{gathered}
$$




\subsection{Background updating}

Initial background is generated by extracting several frames image, background image is constantly updated to gradually close to the true background because of influence of the light, weather and tree swing factors. It is not contain a background update traditional background difference, if background image contains stationary target, or background contains moving target in the initialize background, it is large difference between obtaining background and the true background, so the segmented moving target is inaccurate. In view of the above reasons, the algorithm suitable for background changing is proposed. The specific algorithm is as follows.

If the differential pixel $D^{k-1}(x, y)=0$, the pixel is taken as background, it is that update background is sum of weighting with the current frame and the current background. If the differential pixel $D^{k-1}(x, y)=255$, the background is unchanged. Which the stationary target is removed from the instantaneous background, the specific expression is such as shown in formula (3.5).

$$
B J^{k}(x, y)= \begin{cases}(1-\beta) * B J^{k-1}(x, y)+\beta * J^{k}(x, y) & \text { if } \quad D^{k-1}(x, y)=0 \\ B J^{k-1}(x, y) & \text { if } \quad D^{k-1}(x, y)=255\end{cases}
$$

In formula (7), $B J^{k-1}(x, y)$ is update background, $D^{k-1}(x, y)$ is differential pixel, $J^{k}(x, y)$ is the current background, $\beta$ is weighted value .

The background BJH in the $\mathrm{H}$ space and the background BJI in the I space are obtained by respectively updating each frame using the above algorithm, the accurate segmentation background is provided as threshold segmentation in $\mathrm{H}$ space and I space. in formula (7), $\beta$ is very important, $\beta$ is too small, the background update rate is declined, after updating background does not reflect the instantaneous change of the scene. $\beta$ is too big, the effects of noise is enhanced, the requirements of the hardware processing speed is increased. A better effect is achieved when $\beta$ is $0.1-0.2$.

\section{3 threshold selection}

In this paper, threshold extraction is extracted in $\mathrm{H}$ (chroma) channel and I (grayscale) channel of the HSI space. Threshold extraction method is Otsu method that is an adaptive threshold determination method, Its basic principle is that the image is divided into two parts of the background and target based on its gradation characteristic, and wrong probability is the minimum that some goals is wrongly taken as the background and some background is wrongly taken as the goals. If the variance between the background and objectives is greater, the two parts of the image have the greater difference, the threshold segmentation is more accurate. 
The segmentation threshold of the foreground and background is signed as $T$ for each frame $f(x, y)$ of the video, the proportion of foreground pixels is signed as $\omega_{1}$, and average gray is signed as $\mu_{1}$. the proportion of background pixels is signed as $\omega_{2}$, and average gray is signed as. the total average gray of the image is signed as $\mu$,the on-class variance is signed as $g$.

If each frame $f(x, y)$ of the video has L gray gradation, the numbers of total image pixels are $M$, the numbers of pixels the gray gradation are $i$ and its numbers is $m_{i}$, the occurrence probabilities of each gray value are shown in equation (3.6).

$$
p_{i}=m_{i} / M
$$

If the image is divided into two regions of foreground and background by threshold $T$, the range of background gray gradation is in $O \sim T$, and the range of foreground gray gradation is in $T \sim L-1$. .the probabilities of background and foreground occurred are respectively shown in equation (3.7) and (3.8),the average gray values of background and foreground are respectively shown in equation (3.9) and (3.10), the average gray values of the frame image is shown in equation (3.11).

$$
\begin{aligned}
& p_{b j}=\sum_{i=0}^{T} P_{i} \\
& p_{m b}=\sum_{i=T+1}^{l-1} P_{i} \\
& \omega_{b j}=\sum_{i=0}^{T} i P_{i} / p_{b j} \\
& \omega_{m b}=p_{m b}=\sum_{i=T+1}^{l-1} i P_{i} / m b \\
& \omega=\sum_{i=0}^{L-1} i P_{i}=p_{b j} \omega_{b j}+p_{m b} \omega_{m b}
\end{aligned}
$$

The on-class variance of background and foreground is shown in equation (3.12).

$$
\sigma^{2}=p_{b j}\left(\omega_{b j}-\omega_{0}\right)^{2}+p_{m b}\left(\omega_{m b}-\omega_{0}\right)^{2}
$$

The on-class variance is greater; the difference between background and foreground is larger. The biggest on-class variance is obtained by searching in $[0, \mathrm{~L}-$ 1] using formula (3.13) that is the optimal threshold.

$$
T=\operatorname{Arg} \underset{0 \leq T \leq L-1}{\operatorname{Max}}\left[p_{b j}\left(\omega_{b j}-\omega_{0}\right)^{2}+p_{m b}\left(\omega_{m b}-\omega_{0}\right)^{2}\right]
$$


The segmentation result FIG I' is obtained using segmentation by the optimal threshold in the I-space. The threshold segmentation using Otsu method in the $\mathrm{H}$ Space is the same as I space, the one-dimensional histogram of chroma is generated according to counting up the number of pixels for every chroma value in the space $\mathrm{H}$, it is supposed that there are $\mathrm{M}$ chrominance level in the chromaticity diagram, the threshold values are calculated as shown in equation (3.6) to the formula (3.13). The segmentation result FIG H' is obtained using segmentation by the optimal threshold in the H-space. Finally, $\mathrm{R}$ 'is obtained by being OR on I 'and H'.

\section{The results and analysis of experimental}

In this paper, the video is collected and perseverated in order to verify the feasibility of the algorithm in different light, different weather and the environment of leaves swing and so on, it is programmed using VC ++ in order to verify the feasibility of the results. We can be seen from the test results that the difference of test results by this algorithm and using the traditional background subtraction method is little in simple background, but in complex environment, the test results detected by the algorithms is more accurate and clear than the test results detected by the traditional background subtraction method. The result Fig2 and Fig3 are results in complex environment by this algorithm and using the traditional background subtraction method.

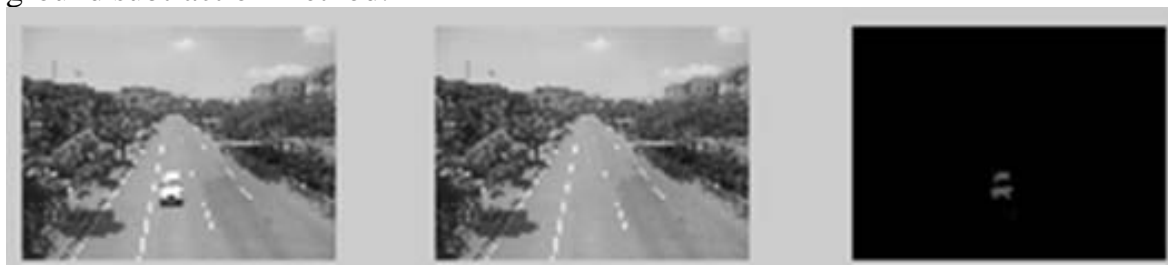
(a) original image
(b) background image
(c) Target detection

Fig 4.1 the algorithm results of this paper

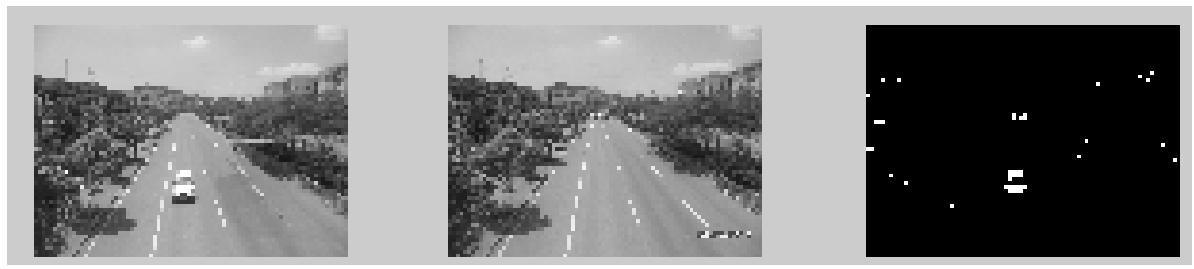

(a) original image

(b) background image

(c) Target detection

Fig 4.2 the algorithm results of background subtraction 


\section{Conclusions}

We can be seen from the test results that the test results detected by the algorithms and he traditional background subtraction method are good in simple background and single video image having single moving object, but in complex environment and especially multi-target environment, the test results detected by the algorithms is better than the test results detected by the traditional background subtraction method, and the algorithms is able to adapt to scene of slowly changing light and weather. The initial background is constantly updated to gradually close to the true background, so the above results can be achieved, and through the detection of the color information, it can effectively reduce the errors that the background is considered to be the target and the target is considered to be the background, the threshold is dynamically determined by the Otsu method, so the image is accurately divided into the target and background, and the accuracy of detection is improved. the algorithm is more complex, the time of detection is relatively long, it needs a relatively high hardware system in practical application, the cost of the system is increased using the algorithms, So the algorithm need be studied and optimized in order to having accurate and practical algorithm.

\section{Acknowledgment}

This work is supported by the science and technology research project of Hubei provincial department of education of China (b20104410).

\section{References}

1.HU W M, TAN T N. A survey on visual surveillance of object motion and behaviors[J].IEEE Transactions on systems, Man and Cybemetics-Part C: Applications and Reviews,2004,34(3):334-352.

2.YoneyamaAkio, Nakajima, asuyuki. Moving object detection from MPEG video stream[J],systems and computers in Japan, 1999,.30(13):1-12.

3.Kutsov R V, Trifonov A P. Detection of a moving object in the image. Journal of Computer and Systems Sciences International,2006,45(3): 459-468.

4.Li-Yeh Chuang;Sheng-Wei Tsai;Catfish Binary Particle Swarm Optimization for Feature Selection[A];Proceedings of International Conference on Machine Learning and Computing (ICMLC 2009) [C];2009.

5.P.Rajkumar;B.Tech;;Motion Detection Based Interactive Surveillance Systems for Mobile Clients[A];Proceedings of International Conference on Information and Network Technology (ICINT 2011) [C];2011.

6.Gary Bradsk i,ADR IAN Kaeh ler.Learning open CV[M].Cambridge: O’ReillyMedia, Inc,2008:12-13..

7.Shaidah Jusoh;HejabM. AlFawareh; Agent-based Knowledge MiningArchitecture[A]; Proceedings of International Conference on Computer Engineering and Applications (ICCEA 2009) $[C] ; 2009$. 